\title{
A PROBABILISTIC APPROACH TO QUANTUM MECHANICS BASED ON 'TOMOGRAMS'
}

\author{
MICHELE CAPONIGRO, STEFANO MANCINI, AND VLADIMIR I. MAN'KO
}

\begin{abstract}
It is usually believed that a picture of Quantum Mechanics in terms of true probabilities cannot be given due to the uncertainty relations. Here we discuss a tomographic approach to quantum states that leads to a probability representation of quantum states. This can be regarded as a classical-like formulation of quantum mechanics which avoids the counterintuitive concepts of wave function and density operator. The relevant concepts of quantum mechanics are then reconsidered and the epistemological implications of such approach discussed.
\end{abstract}

\section{INTRODUCTION}

Since the early days of quantum mechanics, we have been forced to coexist with wave functions [1], therefore with complex probability amplitudes without worrying about their lack of any reasonable physical meaning. One should not ignore, however, that the wave-like properties of quantum objects still raise conceptual problems on whose solutions, a general consensus is far from having been reached [2, 3. A possible way out of this difficulty has been implicitly suggested by Feynmann [4, who has shown that, by dropping the assumption that the probability for an event must always be nonnegative, one can avoid the use of probability amplitudes in quantum mechanics. This proposal goes back to the work by Wigner [5], who first introduced nonpositive pseudoprobabilities to represent quantum mechanics in phase space, and to the Moyal approach to quantum mechanics 6 .

From a conceptual point of view, the elimination of the waves from quantum theory is in line with the procedure inaugurated by Einstein with the elimination of the aether in the theory of electromagnetism [7. Then, quantum mechanics without wave functions has been discussed by several authors 8 .

The predictive character of the wave function led to a statistical description in terms of the density operator [9], which in turn gave rise to speculations about possible analogies with classical statistical mechanics [10. Along this line the phasespace formulation of quantum mechanics [5, 11, 12] provides a means of analyzing quantum-mechanical systems while still employing a classical framework. However, this approach involves counterintuitive notions, like quasi-probabilities, dictated by the uncertainty relations. In fact, as matter of quantum mechanics we cannot see physical objects as they are since the overall back action of any observation cannot be made less than Planck's constant $\hbar$. Instead, we only see the various aspects of the physical objects, like the wave or the particle aspects which depend on the particular kind of observation. In this respect we are really like the prisoners in Plato's famous parable [13] who were chained in a cave and forced to see only the

Date: July 26, 2018. 
shadows of the things outside but not the things as they are. Can we infer the things from their shadows? Tomography is a method for building up a picture of a hidden object from various observable projections (called tomograms or marginals).

Recently, the problem of quantum state measurement, initially posed by Pauli 14, received a lot of attention [15]. The tomographic approach [16 17] to the quantum state of a system has allowed to establish a map between the density operator (or any its representation) and a set of marginal distributions. The latter have all the characteristics of classical probabilities; they are nonnegative, measurable, and normalized. Based on this connection, a classical-like description of a quantum system and its dynamics by means of tomography could be formulated [18, providing a bridge between classical and quantum worlds in the same scenario.

Here, we would analyze such possibility more in details by also considering implications and foundational aspects. Essentially, our aim is to eliminate the hybrid procedure of describing the dynamical evolution of a system, which consists of a first stage, where the theory provides a deterministic evolution of the wave function, followed by a hand-made construction of the physically meaningful probability distributions. If the probabilistic nature of the microscopic phenomena is fundamental, and not simply due to our ignorance, as in classical statistical mechanics, why should it be impossible to describe them in probabilistic terms from the very beginning? On the other hand, the language of probability, suitably adapted to take into account all the relevant constraints, seems to be the only language capable of expressing the fundamental role of chance in nature [19.

The paper is organized as follows. In Sec 2 we introduce the notion of classical states by means of tomograms. Then, in Sec 3 we switch to quantum states. In Sec 4 we discuss the completeness of a set of tomograms. Sec 5 is devoted to the concept of entanglement and Sec 6 to the measurement problem. Finally, Sec 7 point out relations with quantum logic. Sec 8 is for conclusions.

\section{Classical States and Tomograms}

We provide a picture of a classical state by means of tomograms. This enables us to also give a tomographic description of the system dynamics.

2.1. Classical states. The state of a system in classical statistical mechanics is described by a probability distribution function $f(q, p)$ in the phase space $\{q, p\} \equiv$ $\mathbb{R} \times \mathbb{R}$, where $q, p$ are position and momentum coordinate respectively (for the sake of simplicity we are considering a system with one degree of freedom). By definition the function $f(q, p)$ is semidefinite positive and normalized ${ }^{1} \int d q d p f(q, p)=1$. Furthermore,

$$
\int f(q, p) d p, \quad \int f(q, p) d q
$$

represent the marginal probability distributions for position and momentum, respectively, that is the probabilities one can get by measuring observable like position or momentum.

\footnotetext{
${ }^{1}$ Throughout the paper if not specified the integral delimiters must be intended from $-\infty$ to $+\infty$.
} 
Let us now consider a canonical transformation in the phase space $(q, p) \rightarrow$ $(x, y)$, described by

$$
\left[\begin{array}{l}
x \\
y
\end{array}\right]=\mathcal{M}\left[\begin{array}{l}
q \\
p
\end{array}\right], \quad \mathcal{M}=\left[\begin{array}{cc}
\mu & \nu \\
\mu^{\prime} & \nu^{\prime}
\end{array}\right]
$$

where $\mathcal{M}$ is a real symplectic matrix, that is $\mathcal{M S} \mathcal{M}^{T}=\mathcal{I}$ with $\mathcal{S}=\left[\begin{array}{cc}0 & 1 \\ -1 & 0\end{array}\right]$ and $\mathcal{I}$ the $2 \times 2$ identity matrix. The marginal related to the observable $x$ can be written as

$$
w(x)=\int d y f(q(x, y), p(x, y))=\int d q d p f(q, p) \delta(x(q, p)-x) .
$$

Therefore, the marginal $w(x)$ is a real nonnegative, measurable function which is also normalized

$$
\int d x w(x)=\int d q d p f(q, p)=1 .
$$

As a consequence, the function $w$ represent a true probability for the stochastic variable $x$, while it parametrically depends on $\mu, \nu$. Thus, the notation $w(x ; \mu, \nu)$ would be more appropriate.

The variable $x(q, p)$ can be considered from two equivalent points of view. It can be interpreted as a canonically transformed position which is a linear combination of position and momentum in a fixed reference frame in the phase space of the system. Another equivalent interpretation of $x(q, p)$ is that it is a position of the system measured in the rotated and scaled reference frame in the classical phase space of the system.

We use the second interpretation, according to which the real parameters $\mu$ and $\nu$ determine the reference frame in the phase space of the system in which the position is measured. For the position in the transformed reference frame, we get from Eq.(3) the tomography map

$$
w(x ; \mu, \nu)=\frac{1}{2 \pi} \int d q d p d k e^{-i k(x-\mu q-\nu p)} f(q, p) .
$$

Eq.(51) has the inverse [20]

$$
f(q, p)=\frac{1}{4 \pi^{2}} \int d x d \mu d \nu w(x ; \mu, \nu) \exp [i(x-\mu q-\nu p)] .
$$

Example 2.1. The partial case of the canonical transform (2) is a rotation in the phase space for which

$$
\mathcal{M}=\left[\begin{array}{cc}
\cos \theta & \sin \theta \\
-\sin \theta & \cos \theta
\end{array}\right] .
$$

By introducing the notation for the marginal distribution of the rotated position $w(x ; \theta)$ we get, in view of (5),

$$
\begin{aligned}
w(x ; \theta) & =\frac{1}{2 \pi} \int d q d p d k e^{-i k(x-q \cos \theta-p \sin \theta)} f(q, p) \\
& =\int d y f(x \cos \theta-y \sin \theta, x \sin \theta+y \cos \theta) .
\end{aligned}
$$

which is a Radon transform 21]. Its inverse reads

$$
f(q, p)=\frac{1}{4 \pi^{2}} \int d k|k| \int_{0}^{\pi} d \theta \int d x w(x ; \theta) e^{i k(x-q \cos \theta-p \sin \theta)} .
$$

In classical statistical mechanics, the admissible marginal distributions of Eq. (6) always satisfy the condition that $f(q, p)$ is a nonnegative function. 
We have shown that instead of the distribution function $f(q, p)$ the state of the classical system in the framework of classical statistical mechanics can be represented by the marginal distribution function $w(x ; \mu, \nu)$ (intended as the entire set of marginals-probabilities on $x$ corresponding to all possible values of parameters $\mu$ and $\nu)$. Since the map $f(q, p) \mapsto w(x ; \mu, \nu)$ is invertible [20, the information contained in the distribution function $f(q, p)$ is equivalent to the information contained in the marginal distribution $w(x ; \mu, \nu)$.

Example 2.2. If one introduces the distribution function

$$
f(q, p)=\delta\left(q-q_{0}\right) \delta\left(p-p_{0}\right),
$$

which describes a particle with fixed position $q_{0}$ and momentum $p_{0}$, the marginal distribution takes the form

$$
w(x ; \mu, \nu)=\delta\left(x-\mu q_{0}-\nu p_{0}\right)
$$

or

$$
w(x ; \theta)=\delta\left(x-q_{0} \cos \theta-p_{0} \sin \theta\right) .
$$

For classical statistical mechanics, the discussed tomography map connects the positive distributions, and in this context our understanding of the notion of the classical state for systems with fluctuations is unchanged.

2.2. Classical dynamics. The evolution equation for the classical distribution function $f(q, p, t)$ of a particle with unit mass and potential $U(q)$, reads [20]

$$
\partial_{t} f+p \partial_{q} f-\partial_{q} U(q) \partial_{p} f=0
$$

where $\partial_{\bullet}$ indicates the partial derivatives with respect to the variable $\bullet$.

As consequence of the map between $f$ and $w$ developed in the preceeding section, Eq. (13) can be rewritten for time dependent marginal distribution, $w(x ; \mu, \nu, t)$, as

$$
\partial_{t} w-\mu \partial_{\nu} w-\partial_{q} U(\widetilde{q})\left[\nu \partial_{x} w\right]=0,
$$

where the argument of the function $\partial_{q} U$ is replaced by the operator $\widetilde{q}=-\partial_{x}^{-1} \partial_{\mu}$ with $\partial_{\bullet}^{-1} \equiv \int d \bullet$.

Example 2.3. For a harmonic oscillator with unit frequency and potential energy $U(q)=q^{2} / 2$, Eq. (14) becomes very simple, that is

$$
\partial_{t} w-\mu \partial_{\nu} w+\nu \partial_{\mu} w=0 .
$$

Then, the initial distribution 10 takes the form

$$
f(q, p, t)=\delta\left(p-p_{0}\right) \delta\left(q-t p-q_{0}\right),
$$

and the initial marginal distribution (11) becomes

$$
w(x ; \mu, \nu, t)=\delta\left(x-\mu t p_{0}-\mu q_{0}-\nu p_{0}\right) .
$$

\section{Quantum States And Tomograms}

We now straightforward extend the previous approach to the quantum domain.

3.1. Quantum States. In quantum mechanics, a representation of the state in phase space $\{q, p\} \equiv \mathbb{R} \times \mathbb{R}$ cannot be given in terms of probability distributions due to the uncertainty principle which forbids a joint measurement of position and momentum operators $\hat{q}, \hat{p}$. The state can be represented through a quasiprobability function like the Wigner function $W(q, p)$ [5], which plays the same role of the distribution function $f(q, p)$ in the calssical domain. Let us consider the symplectic transformation $\mathcal{M}$ acting on operators vector

$$
\left[\begin{array}{l}
\hat{x} \\
\hat{y}
\end{array}\right]=\mathcal{M}\left[\begin{array}{l}
\hat{q} \\
\hat{p}
\end{array}\right] .
$$


Then, the analogous of Eq.(5) now reads

$$
w(x ; \mu, \nu)=\frac{1}{(2 \pi)^{2}} \int d k d q d p \exp [-i k(x-\mu q-\nu p)] W(q, p) .
$$

We use the same notation as in the classical case. Again, the physical meaning of the parameters $\mu$ and $\nu$ is that they describe an ensemble of rotated and scaled reference frames in which the position $\hat{x}$ is measured. For $\mu=\cos \theta$ and $\nu=\sin \theta$, the marginal distribution (19) is the distribution for the homodyne-output variable used in optical tomography [17. Formula (19) can be inverted and the Wigner function of the state can be expressed in terms of the marginal distribution [18] :

$$
W(q, p)=\frac{1}{2 \pi} \int d \mu d \nu d x w(x ; \mu, \nu) \exp [-i(\mu q+\nu p-x)] .
$$

Since the Wigner function determines completely the quantum state of a system and, on the other hand, this function itself is completely determined by the marginal distribution, one can understand the notion of the quantum state in terms of the classical marginal distribution for squeezed and rotated quadrature. So, we say that the quantum state is given if the position probability distribution $w(x ; \mu, \nu)$ in an ensemble of rotated and squeezed reference frames in the classical phase space is given.

The description of quantum states by the probability function gives the possibility to formulate quantum mechanics without using the wave function or density matrix. These ingredients of the quantum theory can be considered as objects which are not mandatory ones, since they are not directly accessible, but mere abstractions. The marginal probability distribution function $w(x ; \mu, \nu)$, which can be measured directly, replaces the wave function in the new formulation of quantum mechanics. Since the quantum mechanics formalism is reduced to the formalism of classical probability theory, well-known results of the probability theory can be used to get new results in quantum theory. One can also introduce a notion of distance between quantum states in terms of distance between proabilities [22].

Example 3.1. An example of marginal without classical counterpart is given by Schrödinger cat state [2] for which

$$
\begin{aligned}
w(x ; \theta)= & \frac{1}{2} \exp \left[-\left(q_{0}^{2}+p_{0}^{2}\right)\right]\left\{\omega_{A}(x ; \theta)-\omega_{B}(x ; \theta)-\omega_{B}^{*}(x ; \theta)+\omega_{A}(-x ; \theta)\right\} \\
\omega_{A}(x ; \theta)= & \frac{1}{\sqrt{\pi}} \exp \left[-x^{2}-q_{0}^{2} \cos ^{2} \theta-p_{0}^{2} \sin ^{2} \theta\right. \\
& \left.+2 x q_{0} \cos \theta+2 x p_{0} \sin \theta-2 q_{0} p_{0} \sin \theta \cos \theta\right] \\
\omega_{B}(x ; \theta)= & \frac{1}{\sqrt{\pi}} \exp \left[-x^{2}-q_{0}^{2} \cos ^{2} \theta-p_{0}^{2} \sin ^{2} \theta\right. \\
& \left.-2 i x q_{0} \sin \theta+2 i x p_{0} \cos \theta-2 q_{0} p_{0} \sin \theta \cos \theta\right]
\end{aligned}
$$

From the tomographic approach it is clear that there are some classical marginals (states) that do not allow quantum counterpart due to the uncertainty principle. An example is provided by Eq.(11). On the other hand it is well known that there are quantum states (marginals) that do not allow classical counterpart. An example is provided by Eq. (21). Thus we conclude that classical theory is not properly included into quantum theory. They are rather distinct with only a partial overlap.

Moreover, since some classical tomograms are not admissible in quantum mechanics. they cannot be obtained by the limit procedure $\hbar \rightarrow 0$ from the quantum ones. Thus classical mechanics is not the $\hbar \rightarrow 0$ limit of quantum mechanics, as much as like the semigroup cannot give the group properties [23]. 
3.2. Quantum Dynamics. The Wigner function $W(q, p, t)$ evolution is given by the following equation 24 .

$$
\begin{aligned}
\partial_{t} W & +p \partial_{q} W-\partial_{q} U(q) \partial_{p} W \\
& -\sum_{n=1}^{\infty} \frac{(i \hbar / 2)^{2 n}}{(2 n+1) !} \partial_{q}^{2 n+1} U(q) \partial_{p}^{2 n+1} W=0
\end{aligned}
$$

while the evolution equation for tomograms is 18

$$
\begin{aligned}
\partial_{t} w & -\mu \partial_{\nu} w-\nu \partial_{q} U(q) \partial_{x} w \\
& -2 \nu \sum_{n=1}^{\infty} \frac{(i \nu \hbar / 2)^{2 n}}{(2 n+1) !} \partial_{q}^{2 n+1} U(q) \partial_{x}^{2 n+1} w=0
\end{aligned}
$$

Notice that the first three terms of Eq.(25) give the $\hbar \rightarrow 0$ classical Boltzmann equation.

Example 3.2. For a harmonic oscillator with unit frequency and potential energy $U(q)=q^{2} / 2$, Eq. 25 becomes very simple, that is

$$
\partial_{t} w-\mu \partial_{\nu} w+\nu \partial_{\mu} w=0 .
$$

More generally, quadratic Hamiltonians give the same evolution equations for classical and quantum tomograms.

\section{INFORMATION COMPLETENESS}

The problem of how to achieve a kind of measurement that is "complete" in the sense that it can be used to infer information on all possible (also exclusive) observables dates back to Ref. [25].

Obviously enough, no set of sharp observables can be informationally complete, while a set of (partially) non-commuting unsharp observables can be informationally complete [26]. Actually the problem of determining minimal sets of informationally complete observables can be traced back to a group theoretical problem which is still unsolved in its generality [27. Nonetheless we know that the set of rotated position observables leading to $w(x ; \theta)$ is informationally complete, because there is a one-to-one correspondence between $w(x ; \theta)$ and the quantum state [17. Thus, we may formulate the notion of quantum state as follows. We say that the quantum state is given if the position probability distribution $w(x ; \theta)$ is given for all possible rotated reference frames, i.e. $\forall \theta \in[0, \pi]$. This corresponds to sample the parameter $\theta$ over the set $[0, \pi]$ with uniform probability measure $\mathcal{Q}(\theta) d \theta \equiv(1 / \pi) d \theta$.

What happen if we have partial knowledge about $w(x ; \theta)$, that is if we know only some of the tomograms because the parameter $\theta$ has been sampled with a real distribution $\mathcal{P}$ instead of the ideal one $\mathcal{Q}$ ? We can use the relative entropy between $\mathcal{Q}$ and $\mathcal{P}$ to measure the degree of completeness of information achieved by $\mathcal{P}[33]$

$$
H(\mathcal{P} \| \mathcal{Q}) \equiv-\int_{0}^{\pi} d \theta \mathcal{P}(\theta) \log \frac{\mathcal{Q}(\theta)}{\mathcal{P}(\theta)} .
$$

The information contained in the marginal distributions $w(x ; \mu, \nu)$ is somehow overcomplete. In fact, to determine the quantum state completely, it suffices to give the function for arguments with the constraints $\left(\mu^{2}+\nu^{2}=1\right)$ which corresponds to the scheme $\mu=\cos \theta, \nu=\sin \theta$. In particular, suppose to measure the position in reference frames sampled with a distribution $\mathcal{P}(\mu, \nu)$. If we write $\mu$ and $\nu$ in polar 
coordinate, we know that the minimal set of observables to measure only relies on the angular variable, hence we can consider

$$
\mathcal{P}(\theta)=\int_{-\infty}^{+\infty} d r r \mathcal{P}(\mu=r \cos \theta, \nu=r \sin \theta), \quad \theta \in[0, \pi] .
$$

and use it in Eq. (27). Obiously, if $\mathcal{P}$ is uniform over $\theta$ we get a complete information irrespective of the behavior along $r$.

That informationally complete measurements are relevant for foundations of quantum mechanics as a kind of "standard" for a purely probabilistic description has been pointed out also in Ref.[28].

\section{Entanglement and Scaling transforms}

The generalization of the arguments of Sec.III to multipartite systems with $N$ degrees of freedom leads to tomograms of the kind $w(\mathbf{x} ; \boldsymbol{\mu}, \boldsymbol{\nu})$, where $\mathbf{x}, \boldsymbol{\mu}, \boldsymbol{\nu}$ are $N$-components vectors. Let us define the tomographic dispersion matrix elements,

$$
\sigma_{x_{j} x_{k}}(\boldsymbol{\mu}, \boldsymbol{\nu})=\int d \mathbf{x}\left(x_{j}-\bar{x}_{j}\right)\left(x_{k}-\bar{x}_{k}\right) w(\mathbf{x}, \boldsymbol{\mu}, \boldsymbol{\nu})
$$

where

$$
\bar{x}_{j}=\int d \mathbf{x} x_{j} w(\mathbf{x}, \boldsymbol{\mu}, \boldsymbol{\nu}) .
$$

Then, we can construct the dispersion matrix $\mathcal{V}$ using the following relations:

$$
\begin{array}{lll}
\mathcal{V}_{j k}=\sigma_{x_{j} x_{k}}\left(\boldsymbol{\mu}=\mathbf{1}_{j k}, \boldsymbol{\nu}=\mathbf{0}\right), & & j, k=1, \ldots N \\
\mathcal{V}_{j k}=\sigma_{x_{j} x_{j}}\left(\boldsymbol{\mu}=\mathbf{0}, \boldsymbol{\nu}=\mathbf{1}_{j k}\right), & & j, k=N+1, \ldots 2 N \\
\mathcal{V}_{j k}=\sigma_{x_{j} x_{k}}\left(\boldsymbol{\mu}=\mathbf{1}_{j}, \boldsymbol{\nu}=\mathbf{1}_{k}\right), & & j=1, \ldots N ; k=N+1, \ldots 2 N
\end{array}
$$

with $\mathbf{1}_{j k}$ denoting the vector having $1 \mathrm{~s}$ in $j$ th and $k$ th components and zero elsewhere.

To study the separability (entanglement) we use the partial scaling transform to the system 29]. Starting from the tomogram of a state of the system $w(\mathbf{x} ; \boldsymbol{\mu}, \boldsymbol{\nu})$, we first verify that it satisfies the uncertainty principle by checking that $\mathcal{C}=\mathcal{V}+\frac{i}{2} \mathcal{R} \geq$ 0 , where $\mathcal{R}$ is the canonically invariant block diagonal matrix $\operatorname{diag}(\mathcal{S}, \mathcal{S}, \ldots, \mathcal{S})$.

We can now perform an arbitrary scaling described by the vector $\boldsymbol{\lambda}=\left(\lambda_{1}, \lambda_{2}, \ldots \lambda_{2 N}\right)$ on the tomogram, that is $\mu_{j} \rightarrow \mu_{j} / \lambda_{j}$ and $\nu_{j} \rightarrow \nu_{j} / \lambda_{j}$. This implies a scaling on the dispersion matrix leading to

$$
\mathcal{C}^{\boldsymbol{\lambda}}=\mathcal{V}^{\boldsymbol{\lambda}}+\frac{i}{2} \mathcal{R}
$$

where

$$
\mathcal{V}^{\lambda}=\mathcal{D}_{\lambda} \mathcal{V} \mathcal{D}_{\lambda}
$$

with $\mathcal{D}_{\boldsymbol{\lambda}} \equiv \operatorname{diag}\left(\lambda_{1}, \lambda_{2}, \ldots \lambda_{2 N}\right)$. The $2 N$ real parameters $\left\{\lambda_{j}\right\}$ parameterize the Abelian scaling semi-group and we require that

$$
\left|\lambda_{1} \lambda_{2}\right| \geq 1,\left|\lambda_{3} \lambda_{4}\right| \geq 1, \ldots,\left|\lambda_{2 N-1} \lambda_{2 N}\right| \geq 1 \text {. }
$$

The necessary condition for the separability of the state represented by the tomogram $w(\mathbf{x}, \boldsymbol{\mu}, \boldsymbol{\nu})$ is then

$$
\mathcal{C}^{\lambda} \geq 0
$$

for all allowed choices of $\boldsymbol{\lambda}$. 
Out of the $2 N$ scaling parameters we can always choose one pair, $\left(\lambda_{2 k-1}, \lambda_{2 k}\right)$ such that $\left|\lambda_{2 k-1} \lambda_{k}\right|=1$ using the freedom to choose an overall scale factor that does not affect the positivity of $\mathcal{C}^{\boldsymbol{\lambda}}$. For two-mode systems, the choice $\lambda_{1}=\lambda_{2}=$ $\lambda_{3}=1, \lambda_{4}=\lambda^{-1}$ exhausts all the possibilities.

The scaling transformation is not a canonical transformation and can be thought of as an effective scaling of the Planck's constant. A separable state is not sensitive to such scalings applied to individual sub-systems. The entangled states, on the other hand, are sensitive to such scalings and in many cases this shows up by making $\mathcal{C}^{\boldsymbol{\lambda}}$ negative for certain choices of $\boldsymbol{\lambda}$.

Entanglement is synonymous of nonlocal correlations that can be contrasted with local ones through Bell-like inequalities involving only marginal distributions [30].

\section{Quantum Measurements}

It is known 31 that quantum mechanics is problematic in the sense that it is incomplete and needs the notion of a classical device measuring quantum observables as an important ingredient of the theory. Due to this, one accepts that there exist two worlds: the classical one and the quantum one. In the classical world, the measurements of classical observables are produced by classical devices. In the framework of standard theory, in the quantum world the measurements of quantum observables are produced by classical devices, too. Due to this, the theory of quantum measurements is considered as something very specifically different from classical measurements.

It is psycologically accepted that to understand the physical meaning of a measurement in the classical world is much easier than to understand the physical meaning of analogous measurement in the quantum world. Using the relations of the quantum states in the standard representation and in the classical one (described by classical distributions), one can conclude that complete information on a quantum state is obtained from purely classical measurements of the position of a particle made by classical devices in each reference frame of an ensemble of classical reference frames, which are scaled and rotated in the classical phase space.

These measurements do not need any quantum language if we know how to produce, in the classical world (using the notion of classical position and momentum), reference frames in the classical phase space differing from each other by rotation and scaling of the axis of the reference frame and how to measure only the position of the particle from the viewpoint of these different reference frames.

Thus, we avoid the paradox of the quantum world which requires for its explanation measurements by a classical apparatus accepted in the framework of standard treatment of quantum mechanics. The problem of wave function collapse 31 reduces to the problem of a reduction of the probability distribution which occurs as soon as we "pick" a classical value of the classical random observable in the classical framework. This means that we "solved" the paradox of the wave function collapse reducing it to the problem of standard measurement of a classical random variable used in the probability theory.

Nevertheless, measurement on a reference frame affects the distributions on the others (due to the underlying uncertainty principle), thus the nonlocal character of QM is intrinsically present in a single system and emerges as subtle correlations among distributions of different reference frames. 


\section{Relations With Logic}

The developed approach provide a unified logical framework to approach classical and quantum mechanics as summarized by the following scheme.

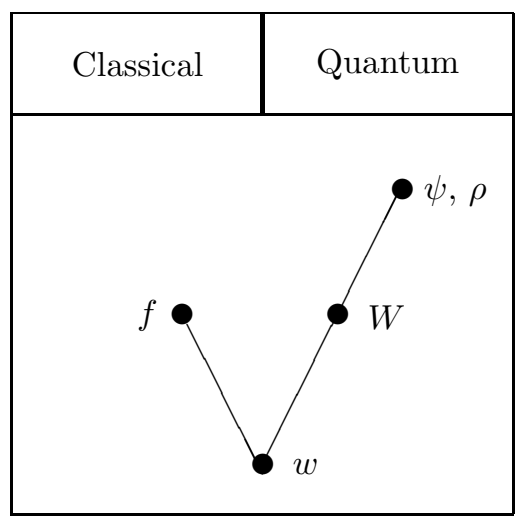

It is worth noticing that the orthodox quantum logic (Birkhoff and von Neumann 34]) used projectors as primitive elemants (propositions). The great problem of quantum logic is that it is so general that it is not useful as model of quantum mechanics. We need more axioms than the assumptions made so as to build a bridge to Hilbert spaces.

Then, Algebraic Approach [34 assumes observables to be primitive and an algebra with natural physical assumtions of linearity, positivity and normalization is constructed. As a consequence there are states which have a place here though not in any classical theory. The aim was to prove the possibility of a quantum theory without Hilbert spaces and to show the possibility of deducing uncertainty principle from an abstract mathematical structure.

Also the tomographic approach does not need of Hilbert space construction. However, observables cannot have a prominent role over states because otherwise phenomenon like entanglement is precluded.

Then, one can consider the Convexity Approach 34 where states are taken as primitive. The principal concept is that of face. This approach has very important consequences for the problem of open systems.

Note that the impossibility of measuring the state of a single system also implies that quantum mechanics cannot only be a theory of states. Then, a StatesObservables Approach was developed by Mackey [35. In this case both states and observables are taken as primitives. Mackey developed a parallelism between quantum mechanics and classical probability calculus: observables are the random variables and the states are probability measures. It was tried to extend the discussion by inserting joint probability measures and conditional expectations: but the first exist only if the observables commute, and the second only if observables have discrete spectrum. Our tomographic approach seems very much in line with Makey's logical construction and circumvent its problems, thus it could be useful to enforce it. 


\section{Conclusions}

We conclude that is possible to cast the standard quantum mechanics into the form in which only positive probabilities are used to describe quantum states and their evolution. A possible disadvantage of the approach proposed is a complicated evolution equation (25), but, perhaps, this is the price one ought to pay for the possibility of describing quantum objects in terms of classical probabilities. Anyway, our arguments can constitute a step further from the Bohr position 36 about the inapplicability of classical modes of description in the quantum domain. In fact, while we belive that quantum mechanics is not classical physics in disguise, we retain (some) classical concepts still applicable against counterintuitive notions like complex statefunctions.

Moreover, our approach can be considered in line with what Wheeler argued: the origin of quantum mechanics structure is to be sought in a theory of observation and observers and meaning, then we would do well to focus our attention not on amplitudes but on quantities which are more directly observable.

However the approach distinguishes from recent information theoretic approaches to quantum mechanics 28, mostly based on epistemic attitude. In such a cases quantum theory is reduced to simple postulates starting from information theoretic principles. This also leads to use probabilities with a 'subjective' character. Moreover, the epistemic point of view cannot be a realist view about the foundations of quantum theory.

The tomographic approach to quantum mechanics is a classic approach, but the probability inferred is not similar with classic epistemic probability. We argue that our assumptions bring us to establish an ontological probability of the physical reality described by classic tomography (as stated in the introduction, if the probabilistic nature of the microscopic phenomena is fundamental, and not simply due to our ignorance, as in classical statistical mechanics, why should it be impossible to describe them in probabilistic terms from the very beginning?). Thus our tomograms assume an ontological character and since they completely characterize the state of a system, also the latter assumes such a character. Due to Primas 32, the distinction between an ensemble interpretation and an individual interpretation is characterized again in terms of the distinction between an epistemic and an ontic interpretation juxtaposing 'description of our knowledge' to 'description of reality'. This distinction seems introducing in the first place problems in the epistemic interpretation as being part of psychology, not physics. Critically, it should be noted that independently of its interpretation, a physical theory is a representation of our knowledge, and therefore is always epistemic (hence bringing us to a Kantian position). Then, it is preferable to avoid the ontic versus epistemic distinction in favour of distinction between individual object versus ensemble.

As we seen, while quantum phenomena require a radical revision of our idea about physical reality, they do not prevent us from accepting a reasonable realistic individual interpretation. Quantum mechanics does not force us to give up realism, but it force us to distinguish carefully between potential and actualized properties. A popular working rule of pragmatic says that an observable has no value before a measurement.This is in contrast to the usual metaphysical commitment of classical mechanics that every observable has a value at all times. This commitment cannot be transferred to quantum mechanics since there is a theorem saying that for a full set of state of a $C^{*}$-algebra, a hypothetical attribution of definite truth values 
to all elements requires that is commutative. However, instead of a positivistic renouncement we can adopt the intrinsic, internally consistent ontological interpretation that at every instant there is a maximal set of truth-definite observables, a truth definite observable possesses a value wheter we know this value or not, is at this stage of the theoretical discussion entirely irrelevant. This point of view corresponds exactly to the usual interpretation of classical point mechanics, where the ontological question of having a value is clearly separated from the entirely different question how to get empirically some information about this value.

Finally, the presented approach can be extended to finite dimensional systems (like spin) 37 and relativistic systems 38. An important analogy with methodology of special relativity arises: It turns out that it is necessary to introduce a consideration of events in the set of moving reference frames in space-time in order to explain relativistic effects, and it is necessary to introduce a consideration of events in the set of rotated and scaled reference frames in the phase space in order to explain the nonrelativistic quantum mechanics in terms of only classical concepts of classical fluctuation theory. But these reference frames are the reference frames in the phase space (not in space-time). Possibly, a combination of these two approaches can be generalized to give a classical description of relativistic quantum mechanics.

\section{REFERENCES}

[1] E. Schrödinger, Ann. der Physik 79, 489 (1926).

[2] E. Schrödinger, Naturwissenshaften 49, 53 (1935).

[3] A. Einstein, B. Podolsky, and N. Rosen, Phys. Rev. 47, 777 (1935).

[4] R. Feynmann, in Quantum Implications, B. J. Hiley and F. D. Peats (Eds.), (Routledge \& Kegan, London, 1987).

[5] E. P. Wigner, Phys. Rev. 40, 749 (1932);

E. P. Wigner, in Perspectives in Quantum Theory, W. Yourgrau and A. van der Merwe (Eds.), (Dover, New York, 1979).

[6] J. E. Moyal, Proc. Cambridge Philos. Soc. 45, 99 (1949).

[7] A. Einstein, H. A. Lorentz, H. Minkowski and H. Weyl, The Principle of Relativity (Dover, New York, 1952).

[8] R. L. Stratonovich, Zh. Eksp. Teor. Fiz. 31, 1012 (1956);

L. Cohen, J. Math Phys. 7, 781 (1966); 17, 1863 (1976);

W. K. Wootters, Found. Phys. 16, 391 (1986);

L. Wang and R. F. O'Connell, Found. Phys. 18, 1023 (1988).

[9] J. von Neumann, Mathematische Grundlagen der Quantenmechanik, (Springer, Berlin, 1932).

[10] E. B. Davies, Comm. Math. Phys. 15, 277 (1969); Quantum Theory of Open Systems, (London, Academic Press, 1976).

[11] K. Husimi, Proc. Phys. Math. Soc. Jpn 23, 264 (1940).

[12] R. J. Glauber, Phys. Rev. Lett. 10, 84 (1963); E. C. G. Sudarshan, Phys. Rev. Lett. 10, 177 (1963).

[13] Plato, Republic, Book VII, in The Loeb Classical Library, L276, Vol.VI (Harvard University Press, Cambridge, 1935).

[14] W. Pauli, Encyclopedia of Physics, (Springer, Berlin, 1958), Vol.5, p.17

[15] Quantum State Preparation and Measurement, J. Mod. Opt. 44, (1997) special issue; D. G. Welsch, W. Vogel and T. Opatrny, Progress in Optics, XXXIX, 63 (1999).

[16] J. Bertrand and P. Bertrand, Found. Phys. 17, 397 (1987).

[17] K. Vogel and H. Risken, Phys. Rev. A 40, 2847 (1989).

[18] S. Mancini, V. I. Man'ko, and P. Tombesi, Phys. Lett. A 213, 1 (1996); S. Mancini, V. I. Man'ko, and P. Tombesi, Found. Phys. 27, 801 (1997).

[19] T. S. Kuhn, The Structure of Scientific Revolutions, (University of Chicago Press, 1970).

[20] O.V. Man'ko and V.I. Man'ko, J.Russ Las.Res. 18, 407 (1997).

[21] J. Radon, Ber. Verh. Sächs. Acad. 69, 269 (1917). 
[22] V. V. Dodonov, O. V. Man'ko, V. I. Man'ko and A. Wünsche, Phys. Scripta 59, 81 (1998).

[23] O. V. Man'ko and V. I. Man'ko, arXiv:quant-ph/0407183

V. I. Man'ko, G. Marmo, A. Simoni, A. Stern and F. Ventriglia, Phys. Lett. A 343, 251 (2005).

[24] C. W. Gardiner, Quantum Noise, (Springer, Berlin, 1991).

[25] E. Prugovecki, Int. J. Th. Phys. 16, 321 (1977).

[26] P. Busch and P. J. Lahti, Ann. der Phys. 47, 369 (1990).

[27] G. Cassinelli, G. M. D'Ariano, E. De Vito and A. Levrero, J. Math. Phys. 41, 7940 (2000).

[28] C. Fuchs, arXiv:quant-ph/0205039

[29] O. V. Man'ko, V. I. Man'ko, G. Marmo, A. Shaji, E. C. G. Sudarshan and F. Zaccaria, arXiv:quant-ph/0502089

[30] S. Mancini, V. I. Man'ko, E. V. Shchukin and P. Tombesi, J. Opt. B 5, 333 (2003);

V. I. Man'ko, E. V. Shchukin and S. Mancini, in preparation.

[31] J. A. Wheeler and W. Zurek, Quantum Theory and Measurement, (Princeton University Press, Princeton, 1983).

[32] H. Primas, Chemistry, Quantum Mechanics, and Reductionism, (Springer-Verlag, Berlin 1983).

[33] A. Wehrl, Rev. Mod. Phys. 50, 221 (1978).

[34] M. L. Dalla Chiara and R. Giuntini, Quantum Logics, (Kluwer, Amsterdam, 2002).

[35] G. Mackey, The Mathematical Foundations of Quantum Mechanics, (Benjamin, New York, 1957).

[36] N. Bohr, Phys. Rev. 48, 696 (1935).

[37] V. I. Man'ko and O. V. Man'ko, JETP 85, 430 (1997).

[38] R.A. Mosna and J. Vaz Jr., Phys. Lett. A 315, 418 (2003)

Physics Department, University of Camerino, I-62032 Camerino, Italy

E-mail address: michele.caponigro@unicam.it

Physics Department, University of Camerino, I-62032 Camerino, Italy

E-mail address: stefano.mancini@unicam.it

P. N. Lebedev Physical Institute, Leninski Prospect 53, Moscow 117924, Russia

E-mail address: manko@sci.lebedev.ru 